\section{Diversidad biocultural y género: Trayectorias productivas de mujeres campesinas de Chiloé}

Biocultural diversity and gender: Productive trajectories of peasant women in Chiloé

\section{DAZMA GuZMÁN*}

\section{Resumen}

Este artículo aborda la situación actual de las mujeres campesinas de la Isla de Chiloé, por medio de la descripción de ocho trayectorias productivas. La base de este trabajo es que las mujeres han liderado hasta hoy la conservación del patrimonio biocultural de su territorio, por lo que representan un actor fundamental para las propuestas de desarrollo orientadas a la sustentabilidad y la identidad cultural. El proceso de investigación se desarrolló durante el año 2013, período durante el cual se realizaron entrevistas en profundidad y observación pasiva

Organización no gubernamental de desarrollo MILPA. Lira 220, Santiago, Región Metropolitana. Correo electrónico: dazma. guzman@gmail.com y participante, dentro de una metodología de carácter etnográfico. Entre los resultados de la investigación, se puede observar que las mujeres campesinas han conformado redes de acción en donde el rescate del conocimiento heredado por las generaciones pasadas se une con las prácticas contemporáneas para crear nuevos proyectos de desarrollo familiar y comunitario que incluyen la conservación del patrimonio biocultural de sus territorios.

Palabras clave: mujeres campesinas, patrimonio biocultural, desarrollo territorial, Chiloé.

\begin{abstract}
This article discusses the current situation of peasant women in Chiloé Island, through the description of eight productive trajectories. The base of this work is that women today lead the conservation of biocultural heritage of their territory, and thus represent a key actor for development proposals aimed at sustainability and cultural identity. The research process, developed in 2013, used the following methods: in-depth interviews, and passive and participant observation within an ethnographic methodology. Among the research findings, it can be seen that currently peasant women have formed networks where local knowledge and biodiversity inherited by past generations meets contemporary practices to create new projects for family, community and regional development, including conservation of biocultural heritage of their territories.
\end{abstract}

Key words: peasant women, biocultural heritage, territorial development, Chiloé. 


\section{Introducción}

Este artículo presenta las trayectorias productivas de ocho mujeres campesinas de la Isla Grande de Chiloé, y ofrece una descripción de las redes y prácticas en las que ellas desarrollan sus actividades. El proceso de investigación se desarrolló durante el año 2013, período durante el cual se realizaron entrevistas en profundidad y observación pasiva y participante, dentro de una metodología de carácter etnográfico.

El contexto en que se desarrolla la investigación muestra a las mujeres campesinas de Chiloé encabezando distintos proyectos de desarrollo familiar y local, en conjunto con distintos actores públicos y privados, en donde la valoración de la identidad cultural es uno de los principales motores para la conservación del patrimonio biocultural en el Archipiélago.

En el planteamiento del problema de estudio, se presentan las dinámicas territoriales que sirven de contexto a los casos considerados, para situar a las mujeres campesinas, en tanto grupo de estudio, dentro de las condiciones que le otorgan relevancia para la investigación y para los procesos de desarrollo del Archipiélago en sí.

Luego, en el marco teórico, al igual que en el metodológico, se recogen los aportes que se han generado desde los estudios de género en las ciencias sociales, en su intersección con el territorio estudiado y el segmento específico de mujeres campesinas. Es decir, se expone el modo en que estas perspectivas pueden aplicarse en términos conceptuales y metodológicos a la realidad específica de Chiloé.

En esta línea, el marco metodológico profundiza en la formulación y aplicación de las herramientas que fueron utilizadas para esta investigación. Además, se agregan antecedentes sobre las características del territorio para contribuir a la comprensión del proceso.

Los casos que se analizaron están conformados por mujeres que se dedican al agroturismo, la artesanía y la producción agroecológica. Cada una de las mujeres que forma parte del universo de esta investigación, vive en diferentes lugares de la Isla Grande de Chiloé y, por ende, han crecido y desarrollado sus actividades en contextos diversos a lo largo de su vida. Por ello se debe considerar que cada una de las mujeres posee características particulares de ocupación, edad, proyectos, historias y trayectorias.

Aun considerando la diversidad interna de los casos abordados, en los resultados se puede observar que sus trayectorias poseen algunos hitos de similitud, desde lo cual se desprende el análisis en que se logra apreciar la importancia que tienen las mujeres liderando iniciativas tanto en su hogar como a nivel comunitario y territorial.

Asimismo, los resultados demuestran cómo las mujeres campesinas en Chiloé han sido protagonistas en la diversificación y valoración del patrimonio biocultural en la isla, generado redes de intercambio y liderando transformaciones en su territorio en pro de un desarrollo local sostenible.

El texto reconoce una tendencia en la que las mujeres protagonizan claramente estas dinámicas de diversificación biológica y cultural, por lo que parece plausible arriesgar que parte de la explicación se encuentra entre las similitudes que existen entre estos casos. Además, se reconoce que el mérito de las mujeres radica tanto en la posición cultural y social que han asumido en el 
proceso de desarrollo, como en la particularidad de cada trayectoria y cada biografía.

\subsection{Problema de estudio}

Por su condición geográfica y política, el Archipiélago de Chiloé se incorporó de manera tardía a los procesos de modernización iniciados en Chile desde la década de los 50 (Centro de Educación y Tecnología [CET] 2006)¹. No fue sino durante la década de 1980 que el territorio de Chiloé experimentó importantes cambios demográficos. Por una parte, esto se debió a la crisis económica que en ese momento atravesaba el país (Ffrench-Davis 2002), lo cual redujo la oferta laboral en Chile continental y, por otra parte, debido al ingreso de diversas industrias acuícolas que generaron enclaves importantes en el territorio gracias a la alta demanda de recursos locales. Entre los principales hitos se encuentran la "Fiebre del loco" (Concholetas concholetas), el "Boom de la merluza" (Merluccius australis), el "Boom de las algas" (Gracilaria spp.) y la salmonicultura, lo que abrió más oportunidades de trabajo dentro de Chiloé. Esto dio como resultado la disminución de la emigración y un aumento en la inmigración desde el centro y sur del país de trabajadores y profesionales que buscaban oportunidades laborales en dichas industrias (Ther y Valderrama 2012; Gajardo y Ther 2011; Macé et al. 2010; Amtmann y Blanco 2001).

Las repercusiones en cuanto a los roles de género establecidos en el territorio de Chiloé se fueron

El proceso de modernización que vivió Chile incluyó aspectos relevantes que influyeron en la vida rural de Chiloé y de otras áreas rurales del país. Específicamente en Chiloé, investigaciones locales y talleres con comunidades campesinas identifican que el proceso de modernización del país, el sistema educativo implementado en las áreas rurales y el efecto del mercado son los factores que explican las dinámicas de cambio locales (CET 2006). dando a medida que la oferta laboral ofrecida por la industria salmonera aumentaba. Para las mujeres esto significó la salida de sus labores cotidianas en el hogar y campo para movilizarse hacia los centros de procesamiento del salmón. A nivel familiar, la inclusión de las mujeres en trabajos asalariados significó un aumento en el ingreso económico familiar, lo cual motivó a otras mujeres a buscar estas fuentes de trabajo para aportar también en sus hogares. Todos los cambios que se generaron en las últimas dos décadas en el territorio han provocado que el aumento en la experiencia de hombres y mujeres haya ido cambiando, por lo tanto esto repercutió en sus estrategias de subsistencia, desde sus actividades tradicionales hacia la industria del salmón y el sector de los servicios.

Como resultado, los campesinos han visto transformada su cultura, ya que tanto mujeres como hombres modificaron las habilidades y el conocimiento adquiridos a través de los medios de subsistencia tradicionales, aplicando habilidades necesarias para el sector acuícola. Al mismo tiempo, se han acentuado cada vez más las diferencias entre los sexos, en cuanto al conocimiento cultural y en particular en lo que se refiere a la continuidad de su transmisión. Esta situación contrasta con la percepción aceptada de una similitud creciente entre las prácticas laborales de hombres y mujeres, en el marco de los procesos de industrialización.

Sin embargo, debido al reciente aumento del desempleo en la industria acuícola, tanto hombres como mujeres han sido obligados a repensar o diversificar sus estrategias de subsistencia familiar. Como parte de estas estrategias se pueden reconocer casos en que se ha apostado por la conservación y recuperación del patrimonio biocultural. 
Como respuesta a la inestabilidad inducida por el modelo industrializador, a partir del siglo XXI se ha consolidado en Chiloé una tendencia opuesta, que apunta a la diversificación económica, cultural y biológica de las actividades campesinas, que es liderada en muchas ocasiones por campesinas y jefas de hogar, por lo que es necesario preguntarse cuál ha sido el rol de las mujeres en la conservación y recuperación del patrimonio biológico y cultural en la estrategia de desarrollo con identidad cultural.

Sin embargo, al margen de la consolidación de estas alternativas, se pudo evidenciar que las dinámicas de homogenización económica, ecológica y cultural promovidas por el modelo de desarrollo neoliberal instaurado por la dictadura militar (1973-1990) y perpetuado por los gobiernos democráticos que le sucedieron hasta la actualidad, pueden erosionar la resiliencia de las comunidades campesinas en Chiloé, por cuanto reduce el repertorio de respuestas disponibles para hacer frente a las necesidades locales, en escenarios cada vez más variables e inciertos (Gómez 2007).

Es así que surge el objetivo de esta investigación, en la que se describen las estrategias de diversificación económica y biocultural de las mujeres campesinas de Chiloé, por medio de la sistematización de los estudios de caso y de la experiencia etnográfica a lo largo de la cual se recabó la información presentada en este artículo.

\section{Marco Teórico Conceptual}

Este artículo se enfocó en hacer un análisis desde la base del liderazgo de las mujeres en proyectos de desarrollo familiar y territorial, que no siguen el patrón establecido por el modelo homogeneizador patriarcal que actualmente es promovido por los grupos de poder económico y político hegemónicos.

Si se atiende al tema de las mujeres campesinas y la problemática que envuelve a este género, son varios los documentos que explican las grandes brechas que separan a muchas mujeres del estándar ideal de desarrollo que buscan conseguir actualmente los gobiernos y organismos internacionales. Como ejemplo de esto, se puede citar el Informe sobre Igualdad de Género y Desarrollo del Banco Mundial (2012), en donde se muestran estadísticas positivas sobre el acceso a la educación, inserción laboral y aumento de la esperanza de vida de las mujeres en estos últimos diez años. Sin embargo, al contrastar estos datos con otros dados reportados por ONU-Mujeres (2013), Hernández (2012), FAO (2011), entre otros, se puede observar que aún existen grandes debilidades en cuanto a la inequidad que viven las mujeres mundialmente, respecto de variables como la falta de oportunidades para cursar carreras técnicas o profesionales, obstáculos legales y tradicionales para que las mujeres tengan la propiedad de la tierra (Lastarria-Cornhiel 2011), posibilidades para optar a créditos que les permitan empezar microempresas o comprar insumos agrícolas (Bórquez 2011), la baja representación política en cargos de elección popular, entre otros (Hernández 2012; Ranaboldo y Solana 2008; Llanos y Sample 2008).

En este contexto, una de las tantas dificultades que deben sobrellevar las mujeres campesinas, es la preferencia de los programas de asistencia técnica por brindar insumos agrícolas y apoyos a los hombres, sin beneficiar a las mujeres con ellos (Bórquez 2011). Según FAO (2011), las mujeres constituyen el $43 \%$ de la fuerza laboral 
agrícola de los países en desarrollo, mientras que en América Latina esa proporción está marcada por un $20 \%$ de participación femenina, en peores condiciones de estabilidad y remuneraciones que la población masculina. De aquí que la planeación de política pública privilegia a los hombres allí donde las mujeres aparecen como un segmento minoritario. Sin embargo, las metodologías y escalas de medición cuantitativa basadas en cifras macro del mercado agrícola, provocan la invisibilización del rol cultural, biológico y económico de las mujeres, y las políticas públicas basadas en dichas cifras tienden, en consecuencia, a prolongar estos desequilibrios (Ruiz y Castro 2011).

Un ejemplo de esto es demostrado por la investigación realizada en Chiloé por Macé et al. (2010), en donde explican que categorías estereotipadas tales como "ama de casa" o "jefe/jefa de hogar", inducen a la población estudiada a responder sobre la base de términos preestablecidos y que muchas veces no aplican a la situación que viven específicamente. Esto termina provocando que las mujeres y sus labores desaparezcan en las estadísticas, generando un vacío de información que es indispensable subsanar para formular políticas y programas de desarrollo a favor de ellas (ONUMujeres 2013; Ranaboldo y Solana 2008).

Por otra parte, si el análisis del desarrollo se realiza considerando que los datos cuantitativos representan solo parcialidades de la realidad, y se opta por analizar cualitativamente los casos que se encuentran fuera de la homogenización que se plantea desde el modelo neoliberal actual, se pueden localizar varios proyectos que sobresalen por construir dinámicas territoriales que se adaptan a las condiciones y potencialidades locales (Sousa Santos 2011;
Cuví et al. 2000), y es ahí donde el paradigma de las mujeres rurales vulnerables cambia y se posicionan como las impulsoras de nuevos proyectos familiares que tienen como base la valoración y conservación del patrimonio agrocultural de su territorio. Lo anterior abre el campo de análisis de las perspectivas de género, ya que se trata de procesos que repercuten positivamente en su entorno creando nuevos espacios y redes para difundir los saberes locales y, con ello, lograr un desarrollo local sostenible que puede ser replicado por otras comunidades (Deere 2011).

Como una de las manifestaciones de este nuevo paradigma, el Centro Latinoamericano para el Desarrollo Rural (RIMISP), inició en el año 2005 el proyecto de Desarrollo Territorial Rural con Identidad Cultural (DTR-IC), que tiene como principal objetivo empoderar a las comunidades locales en torno a su patrimonio biocultural para valorarlo, protegerlo y aprovecharlo económicamente, con el fin de superar la pobreza local. Este patrimonio está constituido por los activos que forman parte de la diversidad biocultural, la cual para este estudio se entenderá como el complejo biológico y cultural que considera genética, biología, agricultura, lingüística, semiótica, cosmovisión y paisajes, cuya composición presente es resultado de un proceso histórico de transformación y diversificación humana. El patrimonio biocultural, por lo tanto, es un reflejo de la capacidad de las comunidades de diferenciarse entre sí, adaptándose a las condiciones particulares del entorno en que se desenvuelven (Toledo y Barrera-Bassols 2008).

Un pilar importante en esta mirada del desarrollo es la heterogeneidad de los territorios, en donde 'la riqueza de lo diverso' se aprovecha 
y potencializa para beneficiar la inclusión social y económica de las comunidades rurales (Ranaboldo y Leiva 2013; Ranaboldo y Schejtman 2009; Fonte y Ranaboldo 2007). Entre las estrategias que reconoce este proyecto como parte de un desarrollo sustentable, junto con la valoración del patrimonio biocultural, está la articulación de actores productivos e institucionales, así como unir las brechas entre lo urbano y rural para facilitar relaciones de intercambio e innovación (Porras y Ranaboldo 2012; Schejtman y Berdegué 2004).

Estas estrategias han generado cambios en las tendencias que representan los procesos de desarrollo en la provincia, siendo estos reflejados en términos particulares en las trayectorias productivas de las familias campesinas. En este sentido, para los fines de este estudio, se entenderá por trayectorias productivas a la expresión temporal de las estrategias asumidas por una persona a lo largo de su vida para proveerse de sus medios de subsistencia, abordando en este caso a un grupo de mujeres campesinas.

\section{Marco Metodológico}

\subsection{Descripción del territorio}

El territorio de Chiloé es un archipiélago formado por una isla grande y más de treinta islas menores, que en su conjunto abarcan una extensión de $9.181,5 \mathrm{~km}^{2}$, las cuales se ubican dentro de la región de Los Lagos, al Sur de Chile (Ministerio del Interior y Seguridad Pública. Gobernación Provincia de Chiloé 2013). Entre las principales características del sistema de producción de la provincia está la integración de distintos ecosistemas: campo, mar y bosque (Venegas 2009).
La población aproximada de la provincia de Chiloé es de 154.766 habitantes, con un porcentaje de ruralidad del $44 \%$ en toda la región (Gobierno Regional de los Lagos 2013). Respecto a la población originaria del archipiélago, se identifica al pueblo Huilliche como el grupo predominante antes de la llegada de los españoles a la zona. Luego, en los siglos que sucedieron, tuvo lugar un proceso de mestizaje que contribuyó a la conformación de una identidad intercultural, que tuvo un correlato en la integración de los sistemas agroculturales indígenas y campesinos europeos que hoy se relacionan con el concepto de la cultura chilota rural. Lo anterior, sin desconocer que en algunas zonas rurales de Chiloé aún se mantiene un fuerte arraigo a la identidad y herencia cultural del pueblo Huilliche, de manera diferenciada pero articulada a la identidad campesina mestiza (CET 2011).

Según la tipología de territorios que propone RIMISP, Chiloé se define como un territorio con dinámicas contradictorias de desarrollo local (Fløysand et al. 2010), en donde la industria de la salmonicultura posee un crecimiento económico alto y se le contraponen varias iniciativas que se basan en la valoración de la identidad cultural, como la tradición campesina e indígena del archipiélago (Bustos 2012; Ranaboldo 2009; Venegas 2009).

\section{Metodología}

El deseo de conocer las trayectorias productivas de las mujeres campesinas en la Isla Grande de Chiloé, surge del interés por analizar algunos proyectos familiares liderados por mujeres que realizan sus labores en el campo en torno a prácticas agroecológicas y 
que buscan rescatar y conservar el patrimonio biocultural del Archipiélago. Asimismo, se consideró seleccionar los casos que estuvieran vinculados con algunas organizaciones que trabajan enfocándose en el desarrollo rural de la isla. Por esta razón, se contactó con el Centro de Educación y Tecnología CET Chiloé, ONG local dedicada al trabajo con comunidades campesinas en el archipiélago y que tiene más de 20 años de experiencia desarrollando prácticas agroecológicas y promocionando la valoración del conocimiento local.

Los casos seleccionados tienen como elemento común a las mujeres, que se destacan por liderar varias actividades en los predios familiares y en su comunidad. Es por ese papel tan importante que cumplen ellas en el contexto local, que se hace necesario sistematizar cada caso para analizar en profundidad las características que los componen y que los diferencian de otros emprendimientos locales que se desarrollan en el contexto de nuevas ruralidades por las que atraviesa el Archipiélago.

En el marco de esta investigación, en donde se buscaba analizar las trayectorias productivas de mujeres campesinas que realizan diversas actividades en la Isla Grande de Chiloé, se vio la necesidad de definir un grupo de estudio en donde los casos que lo conformaran tuvieran una trayectoria de trabajo basada en la identidad cultural y la conservación y valoración de la biodiversidad local. En este sentido, fue importante identificar la vinculación de las experiencias con algunas organizaciones locales abocadas a esta materia, como el CET-Chiloé, y el conjunto de proyectos en el marco de los cuales estos distintos actores se articulan en la actualidad, de los que cabe destacar SIPAM ${ }^{2}$, y el programa de Desarrollo Territorial Rural con Identidad Cultural de RIMISP. Fue así como se pudo concentrar el análisis en tres grandes grupos, todos basados en una diversidad de actividades productivas, pero con énfasis en rubros específicos. De esta manera, se tiene que el grupo lo conforman mujeres que lideran proyectos artesanales, agroecológicos y agroturísticos.

Así fue como durante el año 2013 se realizó el trabajo etnográfico en donde se recopilaron las experiencias contenidas en esta investigación. Las descripciones e interpretaciones que se incluyen se obtuvieron realizando entrevistas en profundidad con ocho mujeres campesinas con contextos familiares y experiencias muy diferentes entre sí. Algunas dedicadas a la artesanía (2), agroecología (3) y agroturismo (3) en sus predios familiares, y también a realizar otras actividades en su comunidad. Estas tres áreas de actividad son, de hecho, centrales desde una perspectiva práctica en los procesos de materialización del DTR-IC en Chiloé, por lo que la intencionalidad de la muestra estuvo orientada también a abordar una descripción representativa en términos cualitativos de dichos ejes de desarrollo familiar y comunitario. 
Imagen 1. Mapa de ubicación de los casos dentro del Archipiélago de Chiloé.

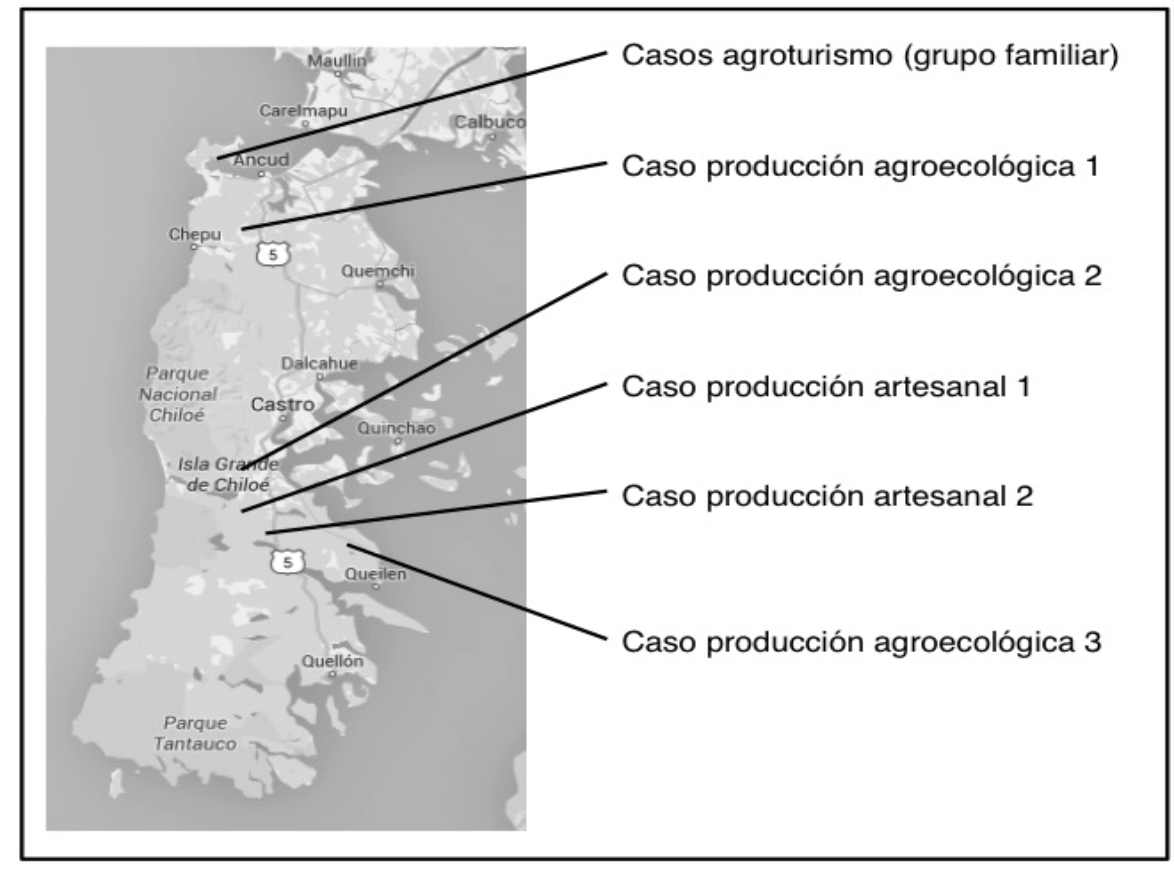

Fuente: Elaboración propia

Junto con las entrevistas, se participó de intercambios de semillas, capacitaciones, giras, etc., lo cual permitió ampliar la descripción de los casos hacia su inserción en redes de acción de diversos niveles territoriales. Como se podrá observar, cada caso ofrece aprendizajes específicos y, por otra parte, el conjunto de ellos ofrece pautas y similitudes que pueden aportar a una comprensión más amplia del tema.

\section{Resultados}

Es necesario empezar por precisar las características que posee cada grupo al cual pertenecen los casos estudiados en profundidad.
Por una parte, las mujeres dedicadas a la artesanía son quienes han aprendido esa actividad por medio de la enseñanza de sus madres $o$ en un ambiente familiar, y han ido innovando en sus técnicas por medio de la formación de agrupaciones con otras mujeres de su localidad y a través de capacitaciones. Dentro de este grupo se recogieron las trayectorias de dos mujeres. La primera de ellas se dedica a la artesanía en lana, teñida con tintes naturales y confecciona prendas de vestir y artículos para el hogar utilizando distintas técnicas de telar. En el segundo caso, se trata de una mujer que elabora artesanía junto a un grupo de mujeres de su comunidad, utilizando madera reciclada que obtiene del bosque que todas manejan. 
El segundo grupo estudiado es el de las mujeres que se dedican a la producción agroecológica, cultivando sus huertos y parte de sus predios de forma orgánica. Es decir, evitan cultivar semillas transgénicas, no utilizan fertilizantes y plaguicidas químicos, y prefieren sembrar cultivos diversos en lugar de dedicarse a un único cultivo. Para este segmento, las mujeres elegidas fueron tres agricultoras que siembran la papa nativa como elemento común, y hortalizas y frutos menores como la frambuesa y arándanos.

Para el tercer grupo, dedicado al agroturismo, se buscó a mujeres que trabajaran en un predio agroecológico y que prestaran un servicio turístico, que además del hospedaje le permitiera al visitante ser parte de las actividades agrícolas que desarrolla la familia cotidianamente. Para este caso se contó con la participación de tres mujeres de diferentes generaciones de una misma familia. A pesar de pertenecer al mismo proyecto agroturístico familiar, las tres mujeres desempeñan diferentes actividades de acuerdo a sus habilidades, y también en algunas ocasiones colaboran todas para brindar un mejor servicio.

De todos los casos que se pudieron conocer, cada uno con relatos muy diferentes entre sí, fue posible distinguir tres hitos esenciales en la vida de las mujeres y que fue el criterio escogido para organizar los resultados en la siguiente sub-sección. El primero, es el impacto e importancia que le otorgan a la transmisión del conocimiento por parte de las madres, abuelas y generaciones anteriores a las que pudieron conocer durante su niñez. Muchas mujeres enfatizaron que su infancia fue una etapa de mucho aprendizaje sobre las labores del campo y que al paso del tiempo estas experiencias aprendidas gatillaron su deseo por quedarse trabajando en sus propios predios. Seguir con la tradición que las generaciones anteriores les enseñaron siendo niñas, las motivó a seguir transmitiendo ese conocimiento a sus hijas y recomendando a estas seguir con el ciclo para evitar su pérdida.

El segundo hito importante abarca el período en que las mujeres salieron de sus hogares por distintos motivos, como empezar una vida con una pareja, el comienzo de sus proyectos personales como opción de diversificación económica del hogar y la formación de nuevas redes relacionadas a su proyecto de vida. Es en este punto en donde las mujeres empezaron a cultivar sus propios huertos, a involucrarse en redes de intercambio para hacerse de más variedades de cultivos, a utilizar de manera independiente los conocimientos que le fueron compartidos por sus madres y abuelas con el fin de aportar económicamente en el hogar, como es el caso de la elaboración de artesanía, la venta de comida tradicional chilota y la siembra de papas nativas para vender en las ferias cercanas, entre otras iniciativas.

Y como tercer hito se encuentra la importancia que le otorgan a la diversificación y conservación del patrimonio agrocultural. Si bien las mujeres relataron que a lo largo de su vida han velado porque sus tradiciones y patrimonio agrícola perdure por varias generaciones más, es en el momento en que se desarrolló la investigación que todas mostraron su convicción hacia la conservación del patrimonio agrocultural, y resaltaron la importancia de la diversificación biológica y económica en su entorno familiar y territorial. Esta convicción es resultado de un proceso doble: tanto personal en la conformación de una experiencia y un discurso 
propio, como colectiva en la articulación de redes junto a otros pares y otros actores como la ONG CET, en donde los valores e inquietudes personales encuentran un espacio propicio para su transmisión, reafirmación, adecuación y promoción.

A continuación se describen con más detalle los tres hitos descritos anteriormente y se incluyen citas textuales que fueron extraídas de las entrevistas realizadas a las mujeres campesinas de Chiloé que formaron parte de esta investigación.

\subsection{Transmisión del conocimiento entre generaciones.}

Muchas de las manifestaciones culturales que se viven actualmente en el archipiélago se han preservado por el liderazgo y compromiso que las mujeres han demostrado tener con las tradiciones de su territorio. El aprendizaje y práctica de diferentes saberes locales como el cultivo del huerto, el manejo del campo y su diversidad de animales y plantas, el proceso de hilado y tejido de artículos con lana, el conocimiento de la gastronomía local y otras tradiciones que hoy perduran en Chiloé han sido transmitidos de generación en generación. En el caso de las mujeres esta transmisión se ha focalizado en aquellos componentes a los que tradicionalmente se han abocado como parte de la distribución de funciones de acuerdo al género al interior del predio. Entre estos componentes se pueden mencionar los huertos, invernaderos, aves de corral y ganadería menor, los que constituyen espacios de diferenciación agrícola y biológica especialmente acentuada. En los relatos realizados por las entrevistadas, se pudo apreciar la importancia que le otorgan a los conocimientos que les fueron compartidos por sus madres y abuelas cuando eran niñas, por ejemplo:

Yo aprendí el cultivo del huerto con mi madre y con mi abuela [...] cultivábamos papa, trigo, avena y en el huerto teníamos betarragas, zanahorias, nabos, lechugas, habas, arvejas, eran casi todas las cosas básicas que todos sembraban y tenían. También con mi abuela aprendí a la ordeña de las vacas y el queso. Ordeñábamos las cabras también para tomar esa leche en el desayuno, ese era nuestro trabajo (Mujer, 62 años, Ancud).

Asimismo, se pudo observar que la transmisión de conocimientos sobre las variedades de plantas para cultivar no se enfocaba sólo en el reconocimiento de las semillas, las variedades que se podían cultivar en el área o la época en la cual debía realizarse la siembra y cosecha, sino que iba más allá. Una parte importante, y que actualmente se continúa transmitiendo, es la forma de preparación de los cultivos en la cocina chilota. Al momento de realizarse un intercambio de semillas, las mujeres comparten no sólo el grano tangible, sino que el acto de regalar o intercambiar una semilla incluye una explicación sobre las condiciones adecuadas en las cuales la planta se desarrolla mejor $y$, además, las distintas formas en que puede prepararse en la cocina.

Un ejemplo de esto sucede con las distintas variedades de papas nativas que se cultivan en la zona. Actualmente hay registradas más de 240 variedades de papa que se cultivan anualmente, muchas de las cuales sirven para platos típicos específicos de Chiloé. Al paso de los años y por distintos factores, como cambios drásticos en el tiempo atmosférico o plagas y una asistencia técnica orientada a la homogenización, se han perdido algunas variedades de papas nativas que antiguamente ascendían con toda seguridad a miles. Muchas de estas son añoradas por lo que significaron en algún momento de la vida 
de las mujeres y sus familias, como se puede observar en la siguiente descripción: "Mi mamá tuvo huerto toda la vida [...] tenía parra, grosellas, plantas medicinales, ajos grandes [...] tenía papas nativas, yo recuerdo que tenía una papa azul larga que la colocaban al horno" (Mujer, 50 años, Chonchi).

Un aspecto importante en cuanto al huerto y al cultivo diversificado en el predio, es lamantención de las especies que las mujeres han tenido desde que iniciaron el trabajo en sus propios campos, y luego la selección de las semillas nuevas para continuar con la siembra de esas variedades. Lo anterior da como resultado un proceso de conservación de semillas sanas, que luego podrán ser intercambiadas para ampliar la diversidad de sus cultivos, obteniendo un ambiente agroecológico en donde se benefician tanto las plantas sembradas como el resto del campo:

Las plantitas que tengo en el huerto, algunas me las dio mi mamá hace muchos años cuando empecé mi propio huerto y otras las tengo porque me las han regalado o en intercambio de semillas. A veces vendo un poco de semillas y otras siempre las guardo para ir sembrando. Así con todas las plantas, los ajos, las papas. [...] Ahora que uno ya siembra con orgánico, con abono de animales es mejor. También tengo plantas medicinales, no tengo muchas, me hace falta ir a buscar más, tal vez en otro intercambio (Mujer, 50 años, Chonchi).

\subsection{Inicio del proyecto propio y creación de redes colaborativas}

A pesar de que las mujeres entrevistadas tenían diferentes edades y que habían vivido experiencias distintas, todas coincidieron en que iniciar un huerto propio o trabajar en un nuevo predio por haberse trasladado ahí luego de casarse, o haber empezado un nuevo proyecto para generar ingresos económicos para el hogar, significó un cambio importante en sus vidas. Esto se debió a que muchas tuvieron que reforzar sus cualidades de liderazgo y hacerse responsables de actividades nuevas que les ayudarían a fortalecer las actividades que habían empezado a realizar de forma más constante. Esta situación se ve ejemplificada en la siguiente cita: "[al salir de la casa de mis padres] yo ya creé mi propio huerto, no para vender sino para el consumo de la casa y para regalar, porque uno si veía que el vecino no tenía una lechuga o un repollo se le daba. Todo se compartía y se regalaba, no se vendía como ahora" (Mujer, 62 años, Ancud).

En algunos casos, la iniciativa y visión de las mujeres les permitió aprovechar las oportunidades que se les presentaban para formar los proyectos que actualmente continúan trabajando y por los cuales son reconocidas dentro de los casos exitosos en su territorio:

\footnotetext{
Hace diez años hace, en el verano siempre pasaba gente acá a preguntar dónde podemos comprar pancito amasado, dónde podemos comprar esto, y no había nada. Entonces yo me di cuenta de que pasaba mucha gente por la carretera, en fines de semana, ya después en pleno verano, entonces ya yo dije por qué no un día no me atrevo y pongo unos letreritos en el cruce de "se vende pan amasado" con una flechita y eso. Así que ya empecé a hacer pan amasado y la gente pasaba y yo vendía. Estaba feliz con eso porque con un par de días ya tenía para comprar un quintal de harina y eso ya era negocio para mí (Mujer, 62 años, Ancud).
}

Si bien las mujeres muchas veces son las que lideran iniciativas de desarrollo local y regional, siguen estando insertas en condiciones estructurales de marginación. Esta condición de desigualdad aparece especialmente dentro de las coordenadas del modelo hegemónico de desarrollo, el que tiende a homogeneizar a la población y a calificar a los grupos sociales de acuerdo a lineamientos que no siempre se 
pueden aplicar a las distintas realidades que se observan en los territorios. Bajo esta premisa de desarrollo modernizador, se invisibiliza la riqueza de lo heterogéneo, obstaculizando los procesos que pueden liderar las mujeres y los grupos sociales que desafían el modelo actual. Esta desigualdad se vive con intensidad en el medio rural, en donde las mujeres, la mayoría de veces, no tienen la titularidad de sus tierras, ya sea por patrones culturales o políticos. También poseen dificultades para optar a créditos que les permitan incorporar nuevas tecnologías a sus predios o proyectos. Esto se puede observar en el relato siguiente, en donde la entrevistada explica las dificultades que tuvieron que vivir con su familia para poder comenzar el negocio que poseen actualmente:

\begin{abstract}
Esto, el predio, visto comercialmente hablando era un predio pobre, devaluado, que nadie hubiera invertido ni una chaucha en este predio. Nadie tiene plata lecheando seis vacas, criando quince ovejas y sembrando diez bolsas de papa, osea, eso es una persona económicamente hablando pobre, visto desde los lineamientos públicos de trabajo, como son las prácticas públicas, como se ve el desarrollo desde la zona central, desde el marginalismo, desde las políticas de desarrollo, nosotros no éramos para nada un bicho interesante po, de hecho no éramos incluyentes para ningún banco, y por eso mismo con mi hermana que tenía un trabajo más o menos estable y yo también, en ese minuto nos encalillamos, entonces pedimos plata y con eso pusimos las bases de esto, como lo material que tuvimos que construir. Entonces así partimos, solas [...] (sic) (Mujer, 43 años, Ancud).
\end{abstract}

En medio del contexto en el que las mujeres se ven marginadas, ellas han buscado formas de organización y redes que les han permitido lograr avances en los proyectos que lideran o en los que son participantes activas. En esos grupos donde encuentran afinidad de intereses, se reúnen con otras mujeres que lideran actividades de otros territorios y en ese encuentro se generan más conocimientos que luego son compartidos con otras y otros miembros de la comunidad. Esto se puede observar en la siguiente afirmación: "en general con la gente con la que yo trabajo o en las redes en las que yo participo, somos mujeres las que andamos en las reuniones, las que vamos a las capacitaciones, las que estamos postulando a proyectos, generalmente somos mujeres" (Mujer, 34 años, Ancud).

Un aspecto importante para las entrevistadas es el aporte económico que generan para el hogar con la venta de los productos cosechados en el huerto o la artesanía y el turismo. Así vemos que las actividades realizadas por ellas forman parte indispensable para mantener la situación económica del hogar, generando un ingreso que les permite continuar con sus actividades mientras atraviesan períodos críticos que pudieran provocar un desequilibrio en sus predios. En el testimonio siguiente observamos el caso de una mujer que, cuando llega la temporada de invierno -en donde la actividad fundamental de su predio, que es la venta de corderos- no se puede realizar, ella sostiene a la familia con las ganancias de la venta de artesanías, vegetales o aves de corral:

Uno colabora con los gastos de la casa con la venta de los tejidos, en invierno que no hay corderos para vender, uno aporta a la casa con el dinero de la venta de tejidos. En diciembre, enero y febrero se venden los corderos y luego el resto de los meses se pone un poco más crítico porque a veces falta la plata. Por eso vendemos los tejidos y algunos de las plantas que cosechamos, papas, semillas, los ajos. También algún animalito como los chanchos, los pavos y las aves que uno cría (sic) (Mujer, 50 años, Chonchi).

\subsection{Diversificación y conservación del patrimonio agrocultural}

Como hemos visto anteriormente, la diversificación biocultural es un proceso 
dinámico, en donde se reúnen distintos actores de todas las edades y de varios territorios. Esto, con el fin de preservar la mayor cantidad de especies de cultivos para que no se pierdan las variedades $\mathrm{y}$, así mismo, poder ampliar las variedades que tiene cada familia. Las personas mayores, muchas veces mujeres, reconocidas entre el grupo por su labor en el campo, y por ende en la conservación del patrimonio biocultural, son las que se han encargado de recolectar las semillas, separarlas para guardar las mejores, sembrarlas y compartirlas con la comunidad. Así se desarrollan los intercambios de semillas, de conocimientos y de tradiciones. Estos intercambios pueden hacerse de forma grupal entre redes que comparten los mismos intereses, como es el caso de los productores orgánicos, o simplemente desarrollarse en una visita a la persona que puede tener una variedad que interesa cultivar. Estas formas de intercambio solidario son una pieza clave para la diversificación de los cultivos en los predios de Chiloé:

Antes uno compraba algunas semillas, bajaba al pueblo
y habían locales que vendían, pero en aquellos años
las semillas eran semillas limpias, las variedades eran
muy productivas y después yo guardaba semillas, por
ejemplo, ahora siguen existiendo las mismas semillas de
las betarragas, zanahorias y otras semillas, entonces uno
trata de seguir usando las mismas semillas (sic). Ahora lo
que hacemos es intercambiar las semillas, siempre con
un grupo de productores orgánicos para tener semillas
limpias (Mujer, 62 años, Ancud).

La diversificación no sólo se puede observar en los huertos chilotes, también se ve en las diferentes variedades de artesanía que se realizan. Con el paso de los años y el apoyo a los grupos de mujeres por medio de capacitaciones por parte de programas del Estado, ONGs o instituciones privadas, se han ido consolidando innovadoras formas de hacer artesanía utilizando materiales propios de la isla y combinando técnicas. Así es como en el siguiente caso, una mujer que reside en la comuna de Chonchi explica la variedad de productos que actualmente hace para la venta en las ferias de Castro y de su comuna.

Ahora hago más variedad [de tejidos], porque antes tejía el poncho y la sabanilla, para abrigos, y hoy día uno hace más variedades. Uno hace chalecos, gorros, medias, ponchos, polainas. Hace 10 años, cuando nos formamos con el grupo de tejedoras, ahí tomamos cursos de croché, tejido a palillos, cursos de teñido con tinte natural, porque en el grupo teñimos con tintes naturales, puras hierbas que encontramos en nuestras casas (sic) (Mujer, 50 años, Chonchi).

Otro factor que se debe destacar es la preocupación de las mujeres por la producción orgánica o sana de sus cultivos. Al utilizar abonos orgánicos extraídos de su propio predio, la combinación de diferentes plantas en las áreas sembradas y su preocupación por el entorno que las rodea, sitúa a las mujeres como un ejemplo de la utilización de prácticas agroecológicas en el área. Al crear ellas mismas un entorno heterogéneo y equilibrado en sus predios, obtienen varios beneficios, entre ellos está que sus cultivos tienen más probabilidades de crecer sanos, libres de plagas $u$ otras enfermedades que normalmente atacan a los cultivos homogéneos:

\begin{abstract}
Me encanta que hoy día se siembre con orgánico, con el abono que uno hace en los corrales. Eso me gusta mucho, porque uno se da cuenta de que con los otros abonos tienen muchos químicos, igual que los "remedios" que le tiran a las papas. Uno escucha que en otros lados que se les pudren las papas, y que las papas tienen otro sabor, en cambio con abono natural de acá del campo se tiene una papa con un sabor distinto, más sano, rico (Mujer, 50 años, Chonchi).
\end{abstract}

Asimismo, se pudo observar que existe entre las entrevistadas y sus familias un sentimiento de profundo compromiso con el entorno, que se refleja en las prácticas que realizan actualmente en sus predios. Es decir, muchas veces dejan 
saber que prefieren la sostenibilidad de los recursos con los que cuentan, que aprovecharlos en este momento sin pensar en las condiciones que tendrán que vivir las generaciones futuras:

Por eso te digo que el uso y abuso de nuestros recursos naturales se puede llevar directamente a la pérdida de toda la identidad de un territorio, la vida de un territorio. En cambio, con prácticas amigables, con prácticas más sanas, que se pueden linear perfectamente desde la cocina porque nosotros desde nuestra cocina, en este espacio que puede parecer pequeño, muy simple hemos lineado nuestras pautas de trabajo [...] como equipo asumimos el compromiso con la tierra, de no dejar nunca de cultivar la tierra, fue un compromiso que asumimos cuando iniciamos este negocio, como familia (Mujer, 43 años, Ancud).

Hemos visto que los casos analizados para este artículo muestran varias características que los hacen únicos dentro del Archipiélago de Chiloé. Es por esto que no se puede generalizar a todas las mujeres de la isla, sino que se debe hacer énfasis en que estos casos fueron estudiados por reunir muchas fortalezas, como la innovación en los productos, colaborar con la conservación del patrimonio cultural y biológico de la isla, articular una gran red de actores locales en pro del desarrollo territorial, y que todas estas características tienen como elemento común la participación activa de las mujeres.

\section{Conclusiones}

Esta investigación ha permitido generar algunos aprendizajes de índole conceptual, metodológica y aplicada. Sobre el primero de ellos, se pudo explorar propuestas conceptuales que permitieran situar el estudio de género $y$, en particular, la figura de las mujeres dentro de la categoría de territorio, uniendo líneas de pensamiento distintas pero compatibles entre sí.
Dentro del abanico de propuestas conceptuales que se manejan hoy día sobre género, esta investigación tuvo que orientarse hacia el estudio de las mujeres rurales y el territorio en donde se desenvuelven. El beneficio de unir estas dos corrientes de estudio radica en que desde la perspectiva de los estudios del territorio se reconoce la importancia de destacar la participación de las mujeres en distintos ámbitos de desarrollo, y sus capacidades o aportes particulares necesarios para entender e impulsar las dinámicas territoriales. Desde la perspectiva de los estudios de género, surge el interés por visibilizar el papel y los esfuerzos que realizan las mujeres, entendiendo que el territorio es un factor fundamental en la comprensión de sus representaciones identitarias y políticas en el contexto general de la sociedad.

Sin embargo, esta unión entre género y territorio aún es incipiente, y aunque se ha nutrido rápidamente de reflexiones teóricas y conceptuales, es posible observar que aún quedan esfuerzos pendientes para proveer al debate de un cuerpo sólido de evidencia y material empírico, lo cual representa una oportunidad importante de investigación. Es en el espacio de esta oportunidad que esta investigación realiza un aporte, entregando la descripción de las trayectorias productivas de mujeres rurales en Chiloé. Si bien este material debe ser complementado con estudios de profundización y metodologías que permitan abordar escalas mayores de descripción, la metodología etnográfica demostró ser una buena oportunidad para seguir ampliando esta línea de estudio mediante la identificación de elementos cualitativos estratégicos, tales como prácticas e hitos comunes en las trayectorias de mujeres o los procesos de construcción de redes e iniciativas a nivel territorial. 
Respecto a este aspecto metodológico, aplicar un diseño de investigación basado en la etnografía permitió llevar a cabo un trabajo de campo flexible que resultó útil para la construcción de las trayectorias productivas de las mujeres campesinas como casos descriptivos. En este sentido, complementar entrevistas en profundidad con la participación en otras actividades habituales de las mujeres permitió abordar una perspectiva de los casos no sólo de manera individual sino también en su articulación con otros actores y sus redes en el territorio. Resultado positivo de ello, fue mantener contacto cotidiano con las mujeres entrevistadas, quienes se mostraron dispuestas a enseñar y colaborar participativamente en el avance de nuevas investigaciones. Esto hizo notar la apertura que tienen las mujeres de los casos estudiados hacia compartir los conocimientos que poseen, elemento clave para poder sistematizar las actividades que realizan y los aprendizajes que les fueron heredados.

Y en el tercer punto sobre el ámbito aplicado, esta investigación logró identificar al menos de manera exploratoria algunos aspectos que pueden resultar críticos para la propuesta y ejecución de planes y programas orientados al Desarrollo Territorial con Identidad Cultural. En este caso se recogieron aquellos elementos de las trayectorias que ayudan a entender el protagonismo de las mujeres en las dinámicas familiares y comunitarias. En este sentido, desde esta investigación se pueden proponer algunas líneas de acción orientadas a la temática de género y territorio, tanto en el ámbito de la investigación como en la política pública.

Una de ellas es que no se considera necesario realizar nuevas intervenciones en territorios con dinámicas y redes ya estructuradas que funcionan en beneficio de la población, sino que se debe hacer el ejercicio de reconocer las capacidades y estrategias que ya se encuentran en acción en el territorio. Habiendo logrado esto, el desafío se encuentra en sistematizar la realidad que viven las mujeres campesinas, resaltando las capacidades que poseen y que los aprendizajes que se obtengan de esas experiencias puedan escalarse a un nivel territorial más extenso. El reconocimiento de estas fortalezas ayudaría a definir a quién y en qué se podrían invertir los recursos, de modo que tengan un efecto sinérgico sobre dinámicas más amplias.

Con respecto al objetivo de esta investigación, se puede decir que fue posible identificar mediante los estudios de caso y el trabajo etnográfico, que las mujeres campesinas de Chiloé sí manejan estrategias de diversificación biológica y cultural, que poseen similitudes entre sí a pesar de que cada mujer se desempeña y especializa de manera específica en torno a sus actividades de agroecología, agroturismo y artesanía.

Esta estrategia tiene al menos dos dimensiones: una personal y otra colectiva. Esto quiere decir que, por una parte, se encuentran las trayectorias personales en las que las mujeres se desempeñan junto a sus familias y en sus predios, realizando actividades en las que se aplican cotidianamente conocimientos, biodiversidad, prácticas y valores que conforman el patrimonio biocultural de las comunidades campesinas. Y por otra parte, se puede observar que junto con estas trayectorias, las mujeres campesinas van articulando redes entre pares y con otros actores, en las cuales dicho patrimonio encuentra oportunidades para ser reconocido, transmitido y fortalecido. Es 
así como los principios de conservación de la diversidad biocultural operan dentro de estas dimensiones, como vehículo de relaciones agroecológicas y sociales en el territorio.

En las trayectorias de vida de las mujeres campesinas participantes del estudio, se identificaron tres hitos principales que representan el proceso de articulación entre las dos dimensiones de la diversificación biocultural señaladas. En primer lugar, se encuentra la formación doméstica y la transmisión del conocimiento y el patrimonio biológico. En segundo lugar, la construcción de un proyecto propio o familiar durante la cual los componentes heredados son adaptados, reorganizados y puestos en práctica. $Y$, finalmente, un proceso creciente de articulación con otros actores del territorio, en donde consolidan sus proyectos posicionándose al interior de redes que están conformadas por otras mujeres que también desarrollan proyectos familiares y por instituciones públicas y privadas. En esta esfera, las mujeres se dan encuentro en instancias de las cuales también participan organizaciones e iniciativas, como la ONG CET, RIMISP y su programa DTR-IC, la ejecución local de SIPAM, Asociaciones Gremiales, redes de turismo, etc., todas las cuales colaboran en el territorio, prestando servicios de capacitación y facilitando la comunicación y la coordinación entre diversos actores de la comunidad y el desarrollo.

Es en estos espacios donde convergen conocimientos heredados de la tradición chilota con las innovaciones presentes, y las mujeres demuestran iniciativa y liderazgo para aplicar lo aprendido y compartirlo, fomentando así la conservación del patrimonio biocultural de su territorio y transformándola en una oportunidad de desarrollo.
Casos como los que se analizaron en esta investigación, demuestran que las mujeres campesinas conforman un agente de vital importancia en sus localidades, por las dinámicas en que se desenvuelven y que fortalecen cotidianamente. De aquí emana el rol de liderazgo de estas mujeres en el desarrollo, esto es, de la especial capacidad que han mostrado para desplegar -dentro y más allá de sus predios-, aquellas estrategias de diversificación biocultural y productiva, de fuerte raigambre tradicional, conectando biodiversidad, conocimiento, prácticas e identidad, con los procesos territoriales que vive Chiloé.

Resulta evidente que las condiciones que se han descrito como comunes a las estrategias de diversificación de las mujeres campesinas, dependen y están específicamente asociadas al entorno de Chiloé, y a la constitución histórica del desarrollo en el territorio. Sin embargo, el hecho de que las trayectorias permitieran identificar algunos principios de acción y de desarrollo que son comunes entre mujeres de un territorio, es una muestra del amplio abanico de posibilidades de investigación existente para distintos lugares de Latinoamérica y el mundo, a partir de la conjunción de las variables de territorio y género. Estas nuevas miradas permiten aportar la descripción de aspectos novedosos tanto de las mujeres como de los territorios, haciendo énfasis en la posición que ellas tienen en la articulación de la base agroecológica y cultural que los sostienen.

Estas miradas, bien podrían tener consecuencias para la práctica del desarrollo territorial en Chiloé y otros lugares del mundo, ya que dan pie a una evaluación completamente distinta del rol de las mujeres en las dinámicas del territorio, por una parte, como una manera de reconocerles 
su liderazgo y su aporte, pero sobre todo como una vía para empezar a pensar y consolidar nuevas formas y mecanismos de desarrollo, más inclusivos, diversos y sustentables.

\section{Bibliografía}

Amtmann, C. y Blanco, G. 2001. "Efectos de la Salmonicultura en las Economías Campesinas de la Región de Los Lagos, Chile". Revista Austral de Ciencias Sociales 5: 93-106.

Banco Mundial. 2012. Informe sobre Igualdad de Género y Desarrollo del Banco Mundial. Washington DC.: Banco Mundial.

Bórquez, R. 2011. Mujeres indígenas, campesinas y su organización por el acceso a la tierra. Seminario Internacional Mujer Rural: Cambios y Persistencias en América Latina. Centro Peruano de Estudios Sociales (CEPES). Lima, Perú.

Bustos, B. 2012. "Brote del virus ISA: crisis ambiental y capacidad de la institucionalidad ambiental para manejar el conflicto". Revista Latinoamericana de Estudios Urbano Regionales -EURE 115: 219-245.

Centro de Educación y Tecnología (CET). 2006. Dinámicas de cambio en Chiloé y su relación con el mundo rural, un proceso en plena evolución. Proyecto Sitios Ingeniosos del Patrimonio Agrícola Mundial (SIPAM Chiloé), patrocinado por FAO (Food Agriculture Organization-ONU). Chiloé, Chile. 17pp.

Cuví, M., Ferraro, E. y Martínez, A. 2000. Discursos sobre Género y ruralidad en el Ecuador, la década de 1990. Quito: CONAMU.

Deere, C. 2011. "Tierra y autonomía económica de la mujer rural: avances y desafíos para la investigación". En Tierra de Mujeres: Reflexiones sobre el acceso de las mujeres rurales a la tierra en América Latina. La Paz: Coalición Internacional para el Acceso a la Tierra y Fundación Tierra.

FAO. 2011. El estado mundial de la agricultura y la alimentación 2010-2011. Las mujeres en la agricultura. Cerrar la brecha de género en aras del desarrollo. Roma: Organización de las Naciones Unidas para la Agricultura y la Alimentación.

Fløysand, A., Barton, J. y Román, A. 2010. "La doble jerarquía del desarrollo económico y gobierno local en Chile: El caso de la salmonicultura y los municipios chilotes". Revista Latinoamericana de Estudios Urbano Regionales -EURE 108: 123-148.

Fonte, M. y Ranaboldo, C. 2007. "Territorios con Identidad Cultural. Perspectivas desde América Latina y la Unión Europea". Revista Opera. Universidad Externado de Colombia, DTR-IC/ RIMISP, Universitá di Napoli. Bogotá, Colombia.

Ffrench-Davis, R. 2002. "Chile, entre el neoliberalismo y el crecimiento con equidad". Revista de economía política 22(4): 88.

Gajardo, C. y Ther, F. 2011. "Saberes y prácticas pesqueroartesanales: cotidianeidades y desarrollo en las caletas de Guabún y Puñihuil, Isla de Chiloé". Chungara, Revista de Antropología Chilena 43(1):589-605.
Gobierno Regional de los Lagos, 2013. Estadísticas: población y superficie. Provincia de Chiloé. Disponible en: http://www. goreloslagos.cl/estadisticas/poblacion_superficie.

Gómez, J. 2007. "Chile: 1990-2007 Una sociedad neoliberal avanzada". Revista de sociología Chile Hoy. Chile 21: 53-78.

Hernández, R. 2012. Nuevas (y viejas) historias sobre las mujeres rurales jóvenes de América Latina. Resultados preliminares del Programa Nuevas Trenzas. Instituto de Estudios Peruano (IEP). Lima, Perú.

Lastarria-Cornhiel, S. 2011. "Las mujeres y el acceso a la tierra comunal en América Latina". En Coalición Internacional para el Acceso a la Tierra y Fundación. Tierra de Mujeres: Reflexiones sobre el acceso de las mujeres rurales a la tierra en América Latina. La Paz, Bolivia.

Llanos, B. y Sample, K. 2008. 30 años de democracia: ¿en la cresta de la ola? Participación política de la mujer en América Latina. Perú: International Institute for Democracy and Electoral Assistance.

Macé, J. et al. 2010. Dinámicas de Sistemas de Género en Chiloé Central, o la Cuadratura de los Ciclos. Documento de Trabajo N 63. Programa Dinámicas Territoriales Rurales. Rimisp, Santiago, Chile.

Ministerio del Interior y Seguridad Pública. Gobernación Provincia de Chiloé. 2013. Información geográfica: Provincia de Chiloé. Disponible en: http://www.gobernacionchiloe.gov.cl/ geografia.html

ONU-Mujeres. 2013. Informe final: Enfoque territorial para el empoderamiento de las mujeres rurales en América Latina y el Caribe. CEPAL, FAO \& RIMISP. Santiago, Chile.

Porras, C. y Ranaboldo, C. 2012. Valuing our Bio-Cultural Riches. Territorial Development with Cultural Identity Project 2005-2011. Quito: Latin American Center for Rural Development (RIMISP).

Ranaboldo, C. y Solana, Y. 2008. Desigualdad de género en la participación política de las mujeres en América Latina y el Caribe. Documento de Trabajo $\mathrm{N}^{\circ} 23$. Programa Dinámicas Territoriales Rurales. Rimisp, Santiago, Chile.

Ranaboldo, C. 2009. "Recorridos de una Mirada Latinoamericana". El Valor del Patrimonio Cultural: Territorios Rurales, Experiencias y Proyecciones Latinoamericanas. IEP/ Rimisp, 13-38.

Ranaboldo, C. y Leiva, F. 2013. La valorización de los activos culturales: Estrategias innovadoras para el empoderamiento de las mujeres rurales jóvenes. Documentos de Trabajo del Programa 
Nuevas Trenzas. Lima: Instituto de Estudios Peruanos (IEP).

Ranaboldo, C. y Schejtman, A. 2009. El valor del patrimonio cultural. Territorios rurales, experiencias y proyecciones latinoamericanas. Lima: IEP-RIMISP.

Ruiz, P. y Castro, M. 2011. La situación de las mujeres rurales en América Latina. Seminario Internacional Mujer Rural: Cambios y Persistencias en América Latina. Lima: Centro Peruano de Estudios Sociales (CEPES).

Schejtman, A. y Berdegué, J. 2004. Desarrollo Territorial Rural. Debates y temas rurales No.1. Santiago de Chile: Centro Latinoamericano para el Desarrollo Rural (Rimisp).

Sousa Santos, B. 2011. "Epistemologías del Sur". Revista Internacional de Filosofía Iberoamericana y Teoría Social 16 (54):
17-39.

Ther, F. y Valderrama, J. 2012. "Dinámicas territoriales en asentamientos de pescadores artesanales: economías, experiencias y conflictos. El caso de Guabún y Puñihuil en la comuna de Ancud, Chiloé". Revista Cultura-Hombre-Sociedad (CUHSO) 22(1): 61-94.

Toledo, V. y Barrera-Bassols, N. 2008. La memoria biocultural. La importancia ecológica de las sabidurías tradicionales. Barcelona: Icaria.

Venegas, C. 2009. "Chiloé: una reserva de patrimonio cultural en Chile". En Ranaboldo, C. y Schejtman, A. (Eds.). El valor del patrimonio cultural: territorios rurales, experiencias y proyecciones Latinoamericanas. Lima: IEP, RIMISP. 